\title{
Simplified Unified Analysis of Switched-RC Passive Mixers, Samplers and $N$-Path Filters using the Adjoint Network
}

\author{
Shanthi Pavan, Senior Member, IEEE and Eric Klumperink, Senior Member, IEEE
}

\begin{abstract}
Recent innovations in software defined CMOS radio transceiver architectures heavily rely on high linearity switchedRC sampler and passive-mixer circuits, driven by digitally programmable multi-phase clocks. Although seemingly simple circuits, the frequency domain analysis of these Linear Periodically Time Variant (LPTV) circuits is often deceptively complex. This paper uses the properties of sampled LPTV systems and the adjoint (inter-reciprocal) network to greatly simplify the analysis of the switched-RC circuit. We first derive the transfer function of the equivalent linear time-invariant filter relating the input to the voltage sampled on the capacitor in the switched-RC kernel. We show how a leakage resistor across the capacitor can easily be addressed using our technique. A signal-flow graph is then developed for the complete continuous-time voltage waveform across the capacitor, and simplified for various operating regions. We finally derive the noise properties of the kernel. The results we derive have largely been reported in prior works, but the use of the adjoint network simplifies the derivation, while also providing circuit insight.
\end{abstract}

\section{INTRODUCTION}

High linearity samplers and passive mixers are being increasingly used in radio transceivers, both for frequency translation in the analog domain, as well as for conversion to and from the discrete-time and/or digital domain [1], [2]. Moreover, passive mixers can be leveraged to implement frequency translated filtering in mixer-first receivers or so called $N$-path filters [3]. As the filter center frequency can be controlled by a digital clock, this allows for flexibly programmable software-defined radio architectures. The target is to replace external surface acoustic wave (SAW) filters by highly integrated SAW-less CMOS implementations, while retaining interference robustness [2]. The removal of SAW filtering increases the receiver's linearity requirements, and has hence fueled research and development in high linearity MOSFET switch-RC circuits. An example of such a circuit is shown in Fig. 1.

Depending on how the outputs are used, the 4-path circuit in Fig. 1 can act as a time-interleaved sampler, a quadrature I/Q mixer or an $N$-path filter. Although the circuit may seem simple, the analysis of its frequency domain transfer function easily becomes involved. To keep the analysis tractable, nonoverlapping clock phases and simplified models for the switches are usually assumed. We briefly review some of the results of prior works, the simplifying assumptions made, and then propose our technique based on the impulse response of the adjoint network.

The transfer function and input impedance of circuits like the one in Fig. 1 excited by a sinewave voltage input can be

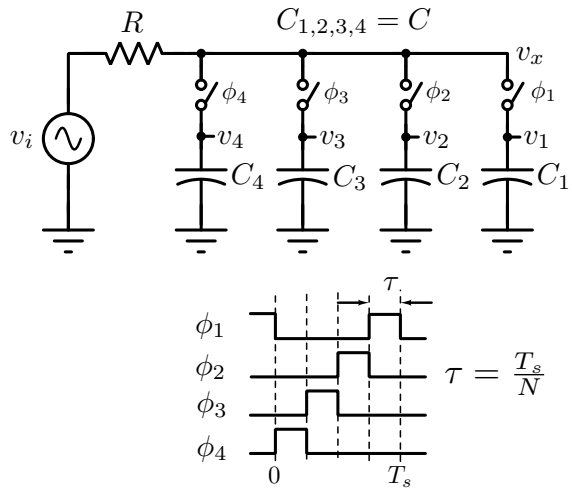

Fig. 1. A 4-path switched-capacitor network that forms the basis for a 4-path passive mixer and the 4-path filter.

approximated assuming $R C \gg \tau$. This has been labeled the "mixing region" approximation [4] or the "passive mixer" region [5], [6]. Under this condition, the voltage on the capacitors becomes virtually constant for an input-frequency close to the switching frequency [7]-[11]. The average power dissipation in the resistor $\mathrm{R}$ can now be evaluated and modeled by an equivalent shunt impedance $Z_{s h}$ in parallel to the capacitor in an Linear Time Invariant (LTI) equivalent model [7], [8]. Baseband resistance in parallel to the capacitor results in an extra dissipation and hence an extra loss resistance.

Another analysis method targeting down-conversion mixer analysis assumes an RF current source with parallel impedance driving four baseband lowpass impedances via 4 switches with $25 \%$ duty cycle [12], [13]. The RF-input frequency $f_{R F}$ is confined to $f_{s} / 2$ and $3 f_{s} / 2$, and the RF-current is approximated in a Fourier series. The RF input voltage is then written in the form of an infinite sum. However, due to the input frequency limitation, signal folding from frequencies higher than $3 f_{s} / 2$ cannot be analyzed.

The above mentioned methods limit the frequency range, and do not predict harmonic responses and signal folding. A large RCtime is assumed, i.e. mixer region operation, which does not cover the sampling region. In contrast, Soer et. al. use the methods of Strom and Signell [14] to provide a unified analysis of samplers and mixers in [4], albeit with lengthy integral calculations to find exact expressions for the harmonic transfer functions of an $N$-path switch-R-C circuit driven by non-overlapping ideal clocks (see for example, the 4-path circuit in Fig. 1). The key design parameters are the input and switching frequency, duty cycle $D=\tau / T_{s}=1 / N$, and the RC time-constant. A sampling 
and mixing region were defined, based on the symmetry found in the noise analysis results around $\Gamma \equiv \tau / R C=2$ (Fig. 12 in [4]). Strongly simplified expressions for the harmonic transfer functions in the sampling and mixing region were derived, which also formed the basis for noise analysis. Later work, building on this analysis derived the filter transfer and noise for $N$-path band-pass [15] and notch filters [16] and an equivalent RLC filter model, gain-boosted $N$-path filters [17], and an $N$-path filter preceded by a series-inductor for low-pass pre-filtering [18]. Such pre-filtering is important, as mixer-first receivers and $N$ path filters show folding of RF-signals around multiples of $f_{s}$ (similar to aliasing in discrete time circuits, albeit with more attenuation).

Whereas the unified analysis of mixing and sampling analyzes the continuous-time output, the unified analysis of [5], [6] focusses on the voltages sampled on the capacitors. The authors of [5], [6] show that the results from [4] in the mixing region can be reproduced assuming ideal reconstruction filtering of the sampled capacitor voltage via a Zero-Order-Hold block $(\mathrm{ZOH})$. Further, their analysis allows for the incorporation a parallel resistor to the capacitor in Fig. 1. Although the analysis is certainly simpler than in [4] and provides valuable insight, it still involves considerable algebra.

This paper, like [5], [6], recognizes the crucial role played by the sampled capacitor voltages on the properties of the $N$ path system. Our analysis then exploits the properties of sampled LPTV networks to derive the results from [5], [6] and [4] in a simpler manner (using lot less algebra). The rest of this paper is organized as follows. In Section II, we summarize the key results on sampled LPTV networks that are relevant to this work [19]. It turns out that the output samples of an LPTV system varying at $f_{s}$, and excited by an input $x(t)$ (when the sampling rate is also $f_{s}$ ) can be thought of as being obtained by exciting an linear time-invariant (LTI) filter by $x(t)$ and sampling its output at $f_{s}$. Further, the impulse response of the equivalent LTI system, denoted by $h_{e q}(t)$, is obtained using the adjoint (or interreciprocal) LPTV network.

Section III uses the results of Section II to derive the expressions for the equivalent LTI filter that relates the input to the voltages held on the capacitors in the $N$-path structure of Fig. 1. We show that the effect of leakage resistance can be incorporated using our technique. We also reflect on the apparent simplicity that characterizes our derivation when compared to prior works.

Section IV then derives a block diagram for the complete waveform of the voltage across one of the capacitors in the $N$ path structure of Fig. 1. We then show the equivalence of our results to those in [4] and [5]. While the block diagram derived in this paper appears more complex compared to that in [4], it turns out that it yields extra insight into the relationship between mixer operation and the sampled capacitor voltage. In addition, the model also allows analysis of $N$-path filters. We then derive simplified expressions for the operation of the $N$-path switch-RC network in the $N$-path filter and passive-mixer modes, and place them in context of prior work. Section V exploits the adjoint impulse-response technique to derive the mean-square noise and noise spectral density of the switched-RC kernel in a simpler manner compared to the frequency-domain method of [4]. Section VI concludes the paper.

\section{LPTV NETwORKS WITH SAMPLED OUTPUTS}

The method of analysis of $N$-path filters and mixers presented in this paper exploits two key properties of sampled LPTV networks [19] summarized below.
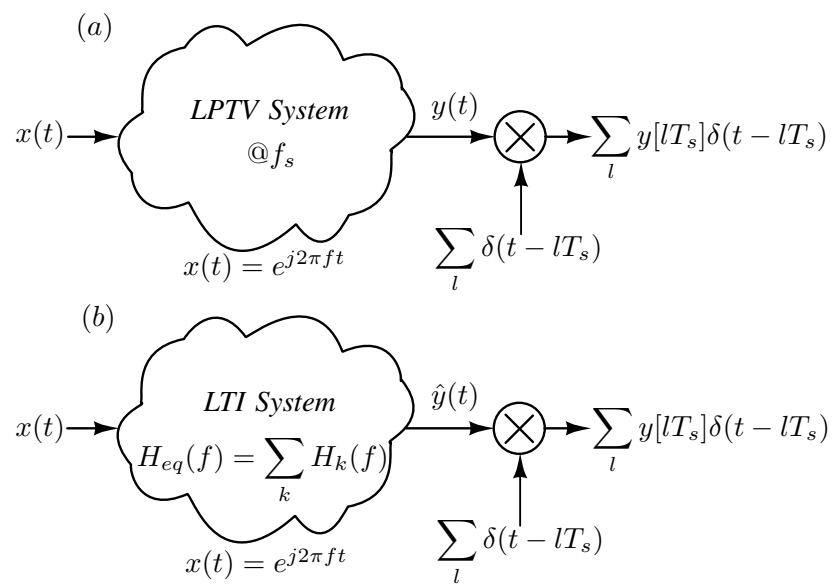

Fig. 2. (a) An LPTV system (varying at a rate $f_{s}$ ) whose output is sampled at $f_{s}$. (b) An LTI system whose output sampled at $f_{s}$ yields the same sequence as the LPTV system above.

Consider an LPTV network varying at a frequency $f_{s}$, as shown in Fig. 2(a). It is excited by a complex exponential $x(t)=$ $e^{j 2 \pi f t}$. The output $y(t)$ is sampled with the period $T_{s} \equiv 1 / f_{s}$ as shown in the figure. Since the system is LPTV, the output consists of frequencies of the form $\left(f+k f_{s}\right)$, where $k$ is an integer. We thus have

$$
y(t)=\sum_{k=-\infty}^{\infty} H_{k}(f) e^{j 2 \pi\left(f+k f_{s}\right) t} .
$$

The samples of $y(t)$ are given by

$y\left(n T_{s}\right)=\sum_{k=-\infty}^{\infty} H_{k}(f) e^{j 2 \pi\left(f+k f_{s}\right) n T_{s}}=e^{j 2 \pi f n T_{s}} \sum_{k=-\infty}^{\infty} H_{k}(f)$.

Consider now the system of Figure 2(b). It is a linear timeinvariant system, whose frequency response $H_{e q}(f)$ is chosen to be $\sum_{k} H_{k}(f)$, where $H_{k}(f)$ are the harmonic transfer functions of the LPTV system of Figure 2(a). If this LTI system is excited by $e^{j 2 \pi f t}$, its output is

$$
\hat{y}(t)=e^{j 2 \pi f t} \sum_{k=-\infty}^{\infty} H_{k}(f) .
$$

When sampled at a rate $f_{s}=1 / T_{s}$, we obtain

$$
\hat{y}\left(n T_{s}\right)=e^{j 2 \pi n f T_{s}} \sum_{k=-\infty}^{\infty} H_{k}(f) .
$$

From (2) and (4), we see that as far as output samples are concerned, an LPTV system whose output is sampled at $f_{s}$ is 
equivalent to an LTI system with output sampled at $f_{s}$. The equivalent LTI filter has a frequency response [19]

$$
H_{e q}(f)=\sum_{k=-\infty}^{\infty} H_{k}(f) .
$$

Since any arbitrary input $x(t)$ can be represented as a sum of complex exponentials via the Fourier transform, it follows that the output samples of an LPTV system (when the sampling rate is the same as that at that the system is varying) can be thought of as being obtained by exciting an LTI filter by $x(t)$ and sampling its output at a rate $f_{s}$. We emphasize that the equivalence holds only for samples, and not necessarily for the waveforms. Referring to Figs. 2(a) and 2(b), we note that $y\left(n T_{s}\right)=\hat{y}\left(n T_{s}\right)$, but $y(t)$ need not equal $\hat{y}(t)$.

How do we determine the transfer function of the equivalent LTI system $H_{e q}(f)$, given an LPTV system? A possible way of doing this is to determine $H_{k}(f)$ of the latter, and then use (5). This, however, leads to tedious algebra.

Fortunately, it turns out that the concept of adjoint (or inter-reciprocal) networks can be used to determine the impulse response $h_{e q}(t)$ corresponding to $H_{e q}(f)$ in only a few steps. The derivation and details are given in [19], we give the results here for the convenience of the reader.
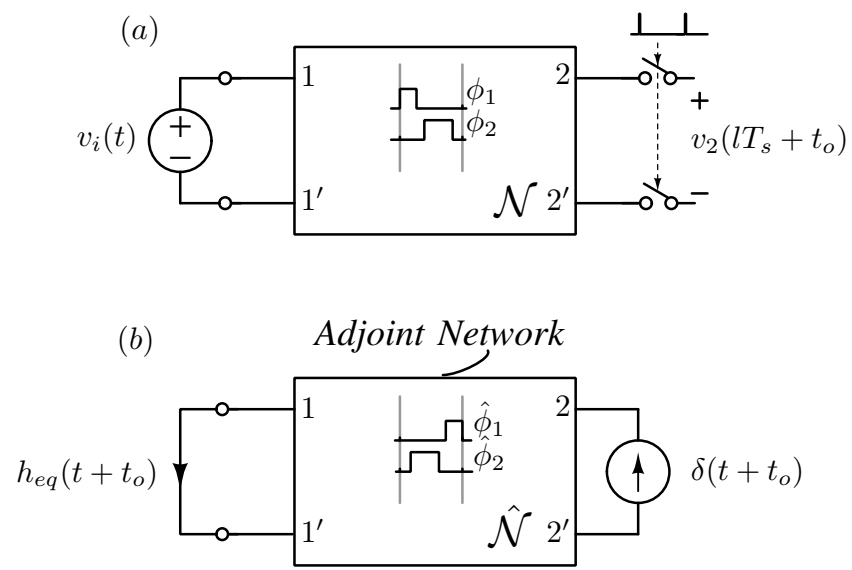

Fig. 3. (a) An LPTV network varying at $f_{s}$, whose output $v_{2}(t)$ is periodically sampled at $f_{s}$. (b) Determining the impulse response $h_{e q}(t)$ of the equivalent LTI filter using the inter-reciprocal (or adjoint) network. An impulse current is injected into the "output" port of the adjoint, and the current in the input port is determined, yielding $h_{e q}(t)$.

Fig. 3(a) shows a 2-port LPTV network $\mathcal{N}$ being excited by a voltage input $v_{i}(t)$. Suppose we are interested in samples of $v_{2}(t)$, taken at a time offset $t_{o}$ from the LPTV sampling clock, namely the sequence $v_{2}\left(l T_{s}+t_{o}\right)$. As discussed previously, one can find an equivalent LTI filter with impulse response $h_{e q}(t)$, which when excited by $v_{i}(t)$, yields an output whose samples are identical to $v_{2}\left(l T_{s}+t_{o}\right)$. $h_{e q}(t)$ can be determined using the adjoint (or interreciprocal) network $\hat{\mathcal{N}}$ corresponding to $\mathcal{N}$, as shown in Fig. 3(b).

Since the output voltage of $\mathcal{N}$ is sampled at $\left(l T_{s}+t_{o}\right)$, the output port of $\hat{\mathcal{N}}$ is excited by a current impulse occurring at $-t_{o}$. It turns out [19] that the resulting current flowing in the input port of $\hat{\mathcal{N}}$ is $h_{e q}\left(t+t_{o}\right)$, as shown in Fig. 3(b). ${ }^{\dagger}$

How does one determine $\hat{\mathcal{N}}$ ? The adjoint network $\hat{\mathcal{N}}$ has the same graph as $\mathcal{N}$, and can be derived from $\mathcal{N}$ by applying the following element-by-element substitution rules shown in Table I [20]-[22]:

1) A branch in $\mathcal{N}$ that is a linear resistor, capacitor, or inductor remains unchanged in $\hat{\mathcal{N}}$.

2) A periodically operated switch in $\mathcal{N}$ controlled by a waveform $\phi(t)$ is replaced in $\hat{\mathcal{N}}$ by a switch that is controlled by $\phi(-t)$.

3) Linear controlled sources in $\mathcal{N}$ are replaced by appropriate linear controlled sources in $\hat{\mathcal{N}}$. For instance, a CCCS in $\mathcal{N}$ is replaced by a VCVS in $\hat{\mathcal{N}}$, with the controlling and controlled ports interchanged, as seen in Table I.

TABLE I

TRANSFORMATIONS OF LINEAR CONTROLLED SOURCES AND PERIODICALLY OPERATED SWITCHES FROM $\mathcal{N}$ TO $\hat{\mathcal{N}}$.

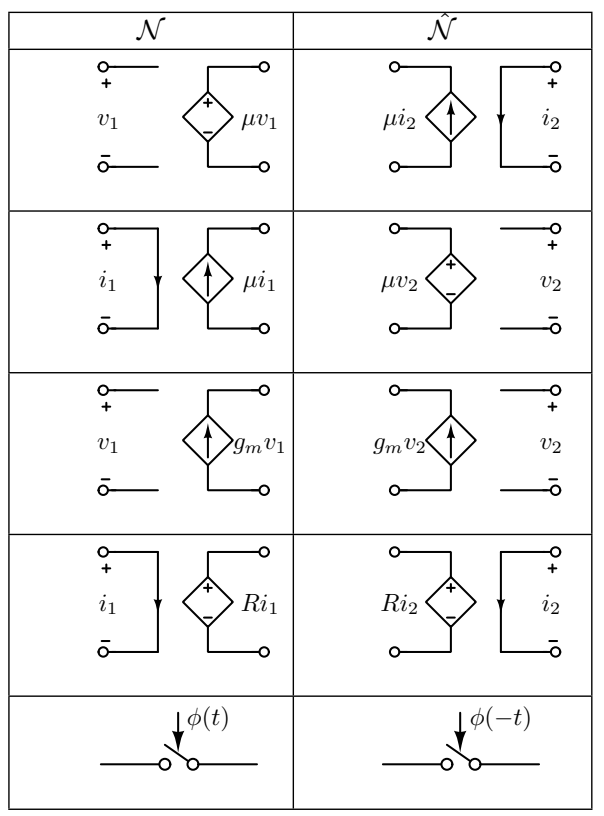

As we show in the next section, the use of the adjoint network greatly simplifies the analysis of the switch-RC $N$-path structure. Adjoint (or inter-reciprocal) networks are well known in circuit theory (see for instance [22]), and form the workhorse for transfer function (.XF), sensitivity and noise analysis in circuit simulators [20], [21]. The adjoint network concept has earlier been applied to the determination of transfer functions and noise in CT $\Delta \Sigma$ Ms [23]-[25], where the input is in continuous-time, while the output is a discrete-time sequence.

\section{The Switch-RC N-Path Circuit}

Fig. 1 shows an $N$-path switched-capacitor network, with $N=4$. While the capacitors are labeled $C_{1, \cdots, 4}$ to aid understanding, they are all equal. Only one of clocks $\phi_{1, \cdots, 4}$ is high

\footnotetext{
${ }^{\dagger}$ Note that exciting output port 2 with a current source and sensing the current at port 1 by adding a short does NOT change the port termination impedance for network $\hat{\mathcal{N}}$ compared to $\mathcal{N}$. Similarly a current output will be excited by a voltage source, while voltage sensing will be used in case of a current driven 2-port, as is done with the analysis of adjoint LTI networks.
} 
at any given time. Each of the waveforms is high for a duration $\tau \equiv T_{s} / N$. Ideal switches are assumed. Clearly, the network is LPTV, varying at $f_{s}$. If $v_{i}(t)=e^{j 2 \pi f t}$, then,

$$
v_{1}(t)=\sum_{l=-\infty}^{\infty} H_{l}(f) e^{j 2 \pi\left(f+l f_{s}\right) t}
$$

where the $H_{l}$ are the harmonic transfer functions. Since the capacitors are equal, and the switch controls are delayed versions of one another, it is easy to show that

$$
v_{n}(t)=\sum_{l=-\infty}^{\infty} H_{l}(f) e^{j \frac{2 \pi(n-1) l}{N}} e^{j 2 \pi\left(f+l f_{s}\right) t}, n=1, \cdots, 4 .
$$

As seen above, the magnitudes of the harmonic transfer functions (HTF) to the capacitor voltages are identical as all the $N$ paths have equal component values but only differ in clock-phase. Thus, the HTFs only differ in phase: the $l^{t h}$ HTF of $v_{n}$ undergoes an additional phase shift of $(2 \pi(n-1) / N) l$ radians. With $N=4$, we have 4-phase quadrature signals:

$$
\begin{aligned}
& v_{1}(t)=\cdots+H_{0}(f) e^{j 2 \pi f t}+H_{1}(f) e^{j 2 \pi\left(f+f_{s}\right) t}+\cdots \\
& v_{2}(t)=\cdots+H_{0}(f) e^{j 2 \pi f t}+j H_{1}(f) e^{j 2 \pi\left(f+f_{s}\right) t}+\cdots \\
& v_{3}(t)=\cdots+H_{0}(f) e^{j 2 \pi f t}-H_{1}(f) e^{j 2 \pi\left(f+f_{s}\right) t}+\cdots \\
& v_{4}(t)=\cdots+H_{0}(f) e^{j 2 \pi f t}-j H_{1}(f) e^{j 2 \pi\left(f+f_{s}\right) t}+\cdots
\end{aligned}
$$

With a proper choice of $R$ and $C$, harmonically combining $v_{1, \cdots, 4}$ can result in a frequency-selective, image-reject, harmonic down-conversion mixer [4]. The voltage $v_{x}$, on the other hand, has a frequency-selective characteristic centered around $f_{s} \equiv$ $1 / T_{s}$ and some of its multiples [12], [15].

From the discussion above, we conclude that determining the HTFs from $v_{i}$ to $v_{1}$ is sufficient; the HTFs to the other capacitor voltages can be obtained using phase-rotational symmetry. Further, since only one of the switches in Fig. 1 is on at any time, there is no coupling between the capacitors. This means that analyzing the circuit with one of the switched branches is all that is needed. This is the so called "switched-RC kernel" [4], shown in Fig. 4(a).

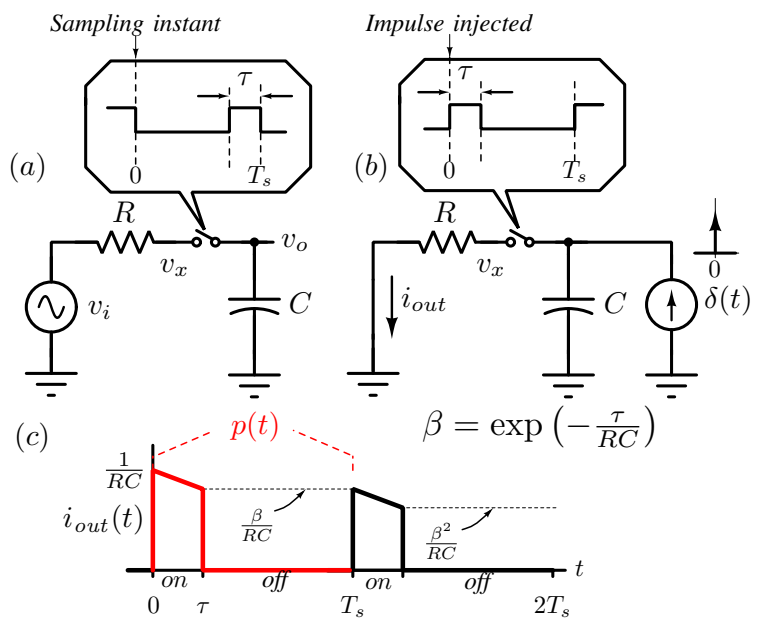

Fig. 4. (a) The switched-RC network. (b) The adjoint (inter-reciprocal) network. The impulse response corresponding to the equivalent LTI filter $H_{e q}(f)$ is the current waveform $i_{\text {out }}$ obtained by injecting at impulse at the "output" port of the adjoint network; i.e., $h_{e q}(t)=i_{\text {out }}(t)$. (c) $i_{\text {out }}(t)$.
We are interested in determining $v_{o}(t)$, which can be separated into two parts; $v_{\text {off }}(t)$ which occurs when the switch is off, and $v_{o n}(t)$, when the switch is turned on. When the switch is off, the capacitor simply holds its voltage. To determine $v_{\text {off }}(t)$, therefore, we would like to know the voltages sampled on the capacitor just before the falling edges of the clock waveform (i.e., at $\left.v_{o}\left(k T_{s}\right)\right)$. In the notation of Fig. $3, t_{o}=0-^{\dagger}$.

The switched-RC circuit is an LPTV network varying at a frequency $f_{s}$, and we are interested in determining $v_{o}\left(k T_{s}\right)$, which is its output sampled at the same frequency as that at which the network is varying. From the discussion in the previous section [19], we know that $v_{o}\left(k T_{s}\right)$ can be thought of as being the samples at the output of a linear time-invariant (LTI) filter, driven by $v_{i}(t)$. Further, [19] shows that the impulse response of this equivalent LTI filter $h_{e q}(t)$, can be readily obtained from the adjoint network (also called the inter-reciprocal network).

The adjoint network corresponding to our example is as shown in Fig. 4(b). Note that the switch-control signal in the original network is reversed in time. The determination of $h_{e q}(t)$ proceeds as follows. The original LPTV network was sampled at zero timing-offset, meaning that we are interested in $v_{o}(t)$ just before $k T_{s}$, which means $t_{o}=0-$. Thus, the "output" port of the adjoint is excited by an impulsive current at $t=0+$ (just after the switch is closed), and the current waveform through the input port is recorded.

Referring to Fig. 4(b), the current impulse causes the capacitor voltage to instantly increase to $v_{x}(0+)=1 / C^{\dagger}$. For $0<t<\tau$, the capacitor discharges through the resistor with a time-constant $R C$, as shown in Fig. $4(\mathrm{c}) . i_{\text {out }}(t)$ during this interval, therefore, is $(1 / R C) \exp (-t / R C)$. Just before $t=\tau$, the capacitor voltage is $v_{x}(\tau-)=(1 / C) \exp (-\tau / R C) \equiv \beta v_{x}(0+)$. For $\tau \leq t<T_{s}$, the switch is open, and $i_{\text {out }}=0$. The voltage across the capacitor does not change during this period. We denote $i_{\text {out }}$ for $0 \leq t<T_{s}$ by $p(t)$, as shown in red in Fig. 4(c).

At $t=T_{s}+$, the switch is closed again, and the capacitor begins to discharge again, but now from a value $\beta v_{x}(0+)$. Note that $h_{e q}(t)=i_{\text {out }}$ is the response to a voltage $v_{x}(0+)$ on the capacitor at $t=0+$. Therefore, the response to a capacitor voltage $\beta v_{x}(0+)$ at $t=T_{s}+$ must be $\beta h_{e q}\left(t-T_{s}\right)$. This is a consequence of linearity and the periodically time-varying nature of the network. Thus, we can write the recursion

$$
h_{e q}(t)=p(t)+\beta h_{e q}\left(t-T_{s}\right) .
$$

In the frequency domain,

$$
H_{e q}(f)=\frac{P(f)}{1-\beta e^{-j 2 \pi f T_{s}}} .
$$

Observing Fig. 4(c) shows that $p(t)$ can be expressed as the difference between two decaying exponentials as follows, where $h(t) \equiv e^{-t / R C} u(t)$ and $u(t)$ denotes the unit-step function.

$$
p(t)=\frac{1}{R C}(h(t)-\beta h(t-\tau)) .
$$

Thus,

$$
P(f)=\frac{1}{1+j 2 \pi f R C}\left(1-\beta e^{-j 2 \pi f \tau}\right) .
$$

\footnotetext{
$\dagger$ Throughout this paper, $t-$ and $t+$ denote the time instants just before and just after $t$ respectively.

${ }^{\dagger}$ The $1 / C$ actually has dimensions of voltage, since the 1 stands for 1 Coulomb.
} 

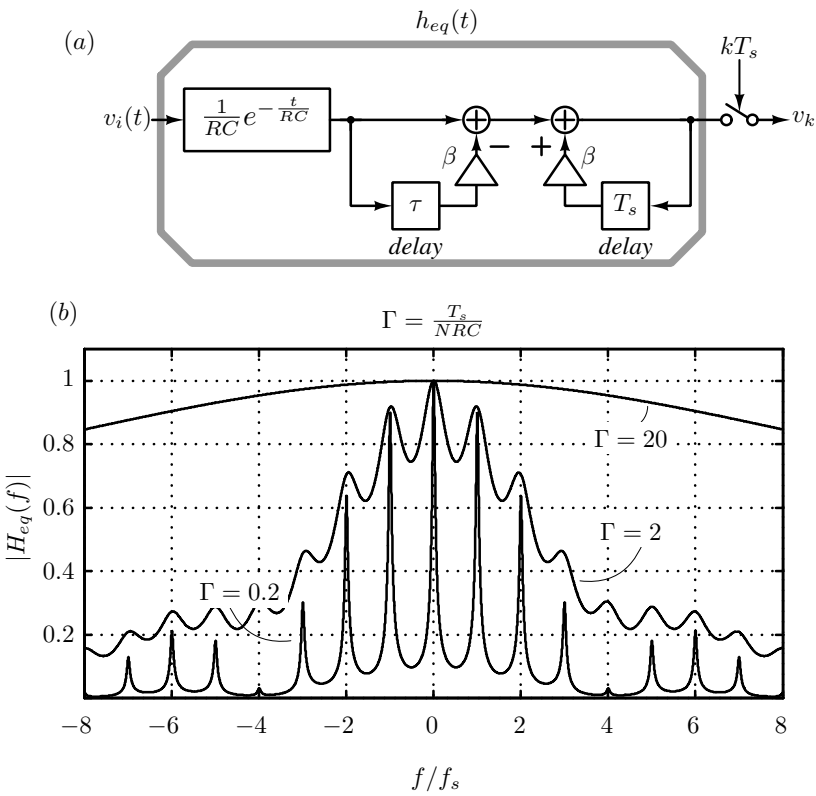

Fig. 5. (a) Block diagram form of the equivalent LTI filter that relates $v_{i}(t)$ to the output samples. (b) Magnitude response of $H_{e q}(f)$ for various values of $\Gamma$. $\Gamma \ll 2$ represents the mixing mode, while $\Gamma \gg 2$ indicates the sampling mode.

Combining (9) and (11), we have

$$
H_{e q}(f)=\frac{1}{(1+j 2 \pi f R C)} \frac{1-\beta e^{-j 2 \pi f \tau}}{1-\beta e^{-j 2 \pi f T_{s}}} .
$$

$H_{e q}(f)$ obtained above is identical to that derived in [5] and [4]. The technique based on the adjoint network that we used above is seen to yield the same result in a much simpler fashion.

It is natural to wonder why the technique above yields (12) in far fewer steps than in prior works. Our explanation is the following. Earlier works arrive at the result that the sampled value of the voltage across the capacitor can be related to the input $v_{i}$ through an LTI filter during the course of analysis. In contrast, we do not discover this while analyzing this particular circuit; we recognize that this property is fundamental to all sampled LPTV networks; we also know that the equivalent impulse response can be found using the adjoint network.

Further, [4], [5] excite the switch-RC network with a sinusoid to determine $H_{e q}$, while we use an impulse current. The "bookkeeping" of the state for the impulse response calculation is simpler for an impulse input, as it "dies" immediately after application. This is in contrast to what happens with a sinewave excitation. This is reflected in the far fewer steps needed to arrive at $H_{e q}(f)$ in our analysis when compared to prior works.

Fig. 5(a) expresses the relationship between the input $v_{i}$ and the sampled output in block diagram form. As discussed earlier, $v_{i}$ can be thought of as being filtered by an LTI system with impulse response $h_{e q}(t)$ (or frequency response $H_{e q}(f)$ ), whose output is sampled at multiples of $k T_{s}$. The LTI system can be thought of as a cascade of a first-order RC filter whose output is delayed, scaled and subtracted from it, followed by a transfer function $1 /\left(1-\beta e^{-j 2 \pi f T_{s}}\right)$. Since this is periodic with a frequency $1 / T_{s}$, the authors of [5] move it beyond the sampler, and interpret it as being a "lossy accumulator" operating on discrete samples. This interpretation runs into difficulties when $C_{1, \cdots, 4}$ begin to interact through parasitic capacitance at $v_{x}$ and/or the series inductance of the source. We therefore, advocate the use of Fig. 5(a) to model the relationship between $v_{i}$ and the voltage sampled on $C_{1}$.

Fig. 5(b) plots the magnitude responses of the equivalent LTI filter for various values of $\Gamma \equiv \tau / R C$. For large $\Gamma(\Gamma \gg 2)$ the second term in (12) is approximately unity, and $H_{e q}(f)$ reduces to the transfer function of a first-order RC lowpass filter. $\Gamma \gg 2$ denotes the "sampling mode" [4] of operation. $\Gamma \ll 2$, on the other hand, results in the "passive-mixer" [5] mode of operation. In this mode, $H_{e q}(f)$ has narrow peaks around multiples of $f_{s}$ (i.e., harmonic selectivity), as seen in Fig. 5(b). Many of these peaks can be eliminated by appropriately combining $v_{1, \cdots, 4}$ in Fig. 1, resulting in mixers with reduced signal and noise folding using the principles of $N$-path operation [26].

(a)

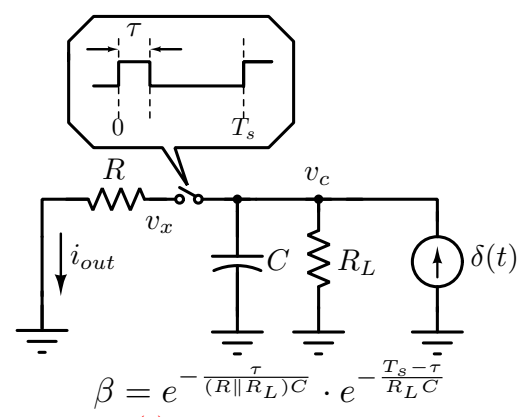

(b)

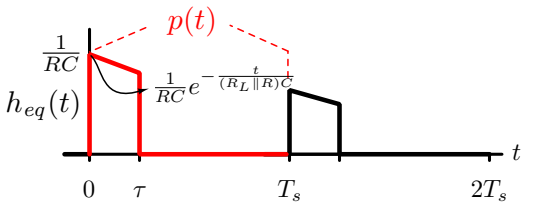

$(c)$

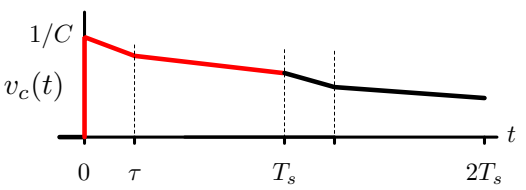

Fig. 6. (a) Adjoint network for the switched-RC kernel with leakage resistor. (b) Current waveform through the resistor. (c) Capacitor voltage waveform.

Using the adjoint method, the effect of a leakage resistance $R_{L}$ in shunt with each of the capacitors in Fig. 1(a) can be analyzed as easily as the "non-leaky" case. Fig. 6(a) shows the adjoint network, where $R_{L}$ denotes the leakage resistor. The initial voltage on the capacitor is $1 / C$, resulting in an initial current $h_{e q}(0+)=1 / R C$. For $0 \leq t \leq \tau$, $h_{e q}(t)$ is an exponentially decaying waveform with time-constant $\left(R \| R_{L}\right) C$.

When the switch is opened at $t=\tau, h_{e q}(t)$ goes to zero. However, the capacitor voltage continues to decay with a timeconstant $R_{L} C$ during the interval $\tau \leq t<T_{s}$, as shown in Fig. 6(c). Thus, the capacitor voltage at $t=T_{s}+$ is given by

$$
v_{x}\left(T_{s}+\right)=\underbrace{e^{-\frac{\tau}{\left(R \| R_{L}\right) C}} \cdot e^{-\frac{\left(T_{s}-\tau\right)}{R_{L} C}}}_{\equiv \beta} v_{x}(0+) \text {. }
$$

Proceeding as we did while deriving (12), it is straightforward to see that with leakage, $\beta$ and $P(f)$ in (9) should be 
replaced by

$$
\beta=e^{-\frac{\tau}{\left(R \| R_{L}\right) C}} \cdot e^{-\frac{\left(T_{s}-\tau\right)}{R_{L} C}}
$$

and

$$
P(f)=\frac{R_{L}}{R_{L}+R} \frac{1-e^{-\frac{\tau}{\left(R \| R_{L}\right) C}} e^{-j 2 \pi f \tau}}{\left(1+j 2 \pi f\left(R \| R_{L}\right) C\right)} .
$$

These equations are the same as those derived in [5]. Setting $R_{L}$ to $\infty$ in the expressions above yields (12), as it should. Again, our analysis to account for leakage resistance is straightforward. Moreover, the time-domain response can provide insight into the effect on frequency selectivity. The leakage resistance results in a reduced peak $H_{0}$, and a lower $Q$ (reduced frequency selectivity), since the impulse response dies out more rapidly. Referring to Figs. 4(b) and 6(b), we see that $\beta$ can physically be interpreted as the fraction of charge remaining on $C$ at the beginning of $t=T_{s}+$.

\section{Complete Output Waveform}

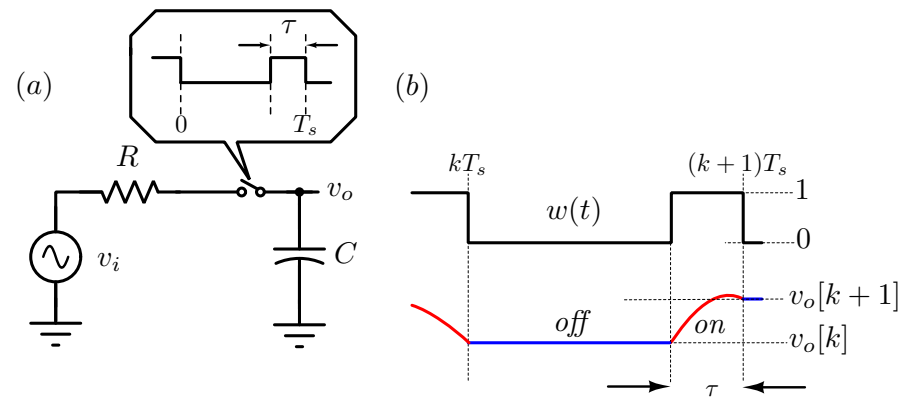

(c)
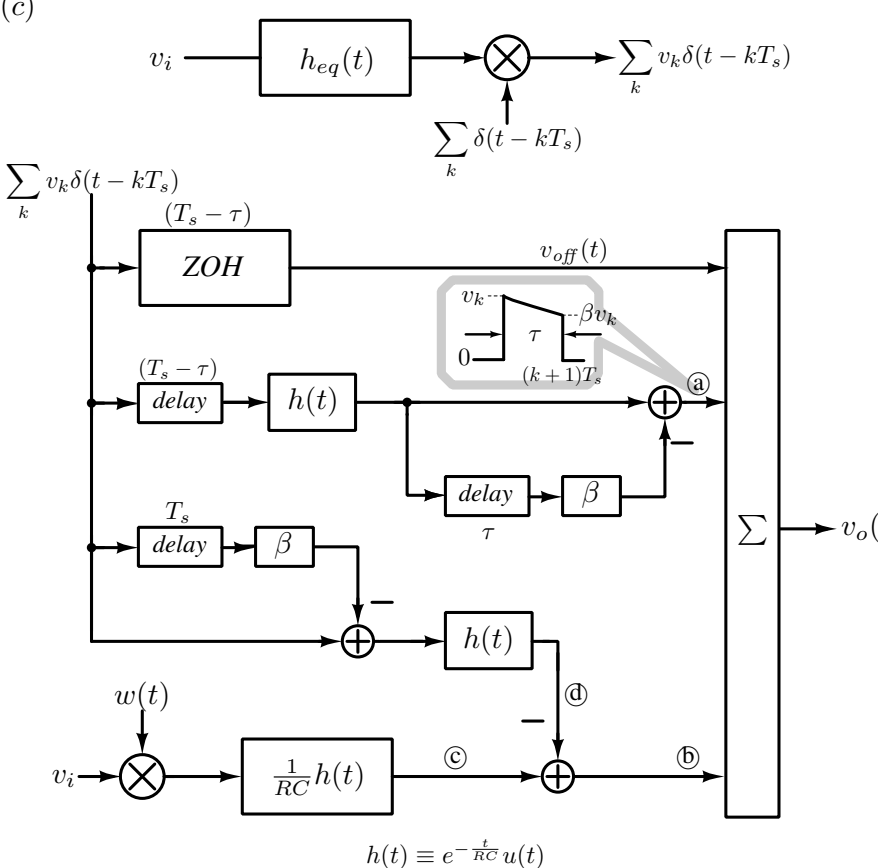

Fig. 7. (a) The switched-RC circuit. (b) Timing and output waveforms. (c) Signal flow diagram.

We now determine the complete output waveform of the switched-RC kernel (without the leakage resistor $R_{L}$ ). The circuit (not the adjoint) is shown in Fig. 7(a). The analysis proceeds as follows.
- We first express the output voltage across the capacitor as the sum of two waveforms: $v_{\text {off }}(t)$ that is non-zero when the switch is open, i.e., the time intervals $k T_{s} \leq t<(k+1) T_{s}-\tau$, and $v_{o n}(t)$, which is non-zero when the switch is closed, i.e., the intervals $(k+1) T_{s}-\tau \leq t<(k+1) T_{s}$, as shown in blue and red in Fig. 7(b) respectively.

- Next, we determine $v_{\text {off }}(t)$. This is very straightforward if we know the voltages sampled on the capacitor at the falling edges of the clock, namely at times $t=k T_{s}-$. We denote the sampled capacitor voltage sequence by $v_{k}$. Then, it is easily seen that $v_{\text {off }}(t)$ is simply the waveform obtained by exciting a zero-order-hold $(\mathrm{ZOH})$ that holds for a duration $\left(T_{s}-\tau\right)$ by the impulse waveform $\sum_{k} v_{k} \delta\left(t-k T_{s}\right)$, as shown in Fig. 7(c).

- We then invoke the results of the previous section to determine $v_{k}$. As we saw there, $v_{k}$ can be thought of as the sampled output of a linear time invariant filter with impulse response $h_{e q}(t)$, when excited by $v_{i n} . h_{e q}(t)$ can be conveniently found using the adjoint network approach.

- Next, we set out to determine $v_{o n}(t)$, which is non-zero only in the intervals $(k+1) T_{s}-\tau<t<(k+1) T_{s}$. Note that $v_{o n}(t)$ must not only depend on the voltage held on the capacitor when the switch is closed $v_{k}$ (this forms the "initial condition") as well as $v_{i n}(t)$. The derivation of $v_{\text {on }}(t)$ proceeds as discussed below.

$v_{o n}(t)$ consists of two parts: a decaying portion due to past inputs and a part due to the current input. The first part is due to "previous history", namely the voltage $v_{k}$ already stored on the capacitor, which will exponentially decay as it discharges through the resistor with a time-constant $R C$. This is modeled by the output of the path labeled $@$ in Fig. 7(c). The explanation for path @ is as follows. When the switch is turned on at $t=$ $(k+1) T_{s}-\tau, v_{o n}(t)$ must be $v_{k}$. This starts to decay exponentially until $t=(k+1) T_{s}$, at which time the switch is opened. This process can be modeled by exciting a filter with impulse response $h(t)-\beta \cdot h(t-\tau)$ with an impulse of strength $v_{k}$ at $t=(k+1) T_{s}-$ $\tau$ (recall that $h(t)=e^{-t / R C} u(t)$ and $\beta=e^{-\tau / R C}$ ). The resulting waveform at $($ is shown in the inset. Fig. 8 shows the waveforms that comprise $v_{o n}(t)$ in more detail. $v_{a}$ (in blue), which is nonzero only when the switch is closed, shows the exponentially decaying waveform due to path @a. At $t=(k+1) T_{s}-\tau, v_{a}$ jumps from 0 to $v_{k}$. At $t=(k+1) T_{s}-$, it has decayed to $\beta v_{k}$, and goes abruptly to zero at $t=k T_{s}$.

The second part of $v_{o n}(t)$ is due to $v_{i}(t)$ exciting the RC circuit when the switch is closed. This part, in turn, is the sum of two components. The first part is the output due to $v_{i}$ exciting the RC network, and can be modeled by driving a filter with an impulse response $(1 / R C) e^{-t / R C} u(t)=(1 / R C) h(t) u(t)$ with the time-windowed input $w(t) \cdot v_{i}(t)$, shown as the output of path (61). $w(t)$, as shown in Fig. 7(b), is 1 when the switch is closed and 0 otherwise. Fig. 8 shows $v_{i}$ and $w(t) v_{i}$ in gray and black respectively. The voltage $v_{b 1}$ shows the output of path 0 . As seen in the figure, $v_{b 1}$ persists when $w(t) v_{i}=0$ due to the memory of the capacitor.

At $t=(k+1) T_{s}$, however, the output of path (b) in Fig. 7(c) must go to zero, since $v_{o n}(t)$ is zero for 


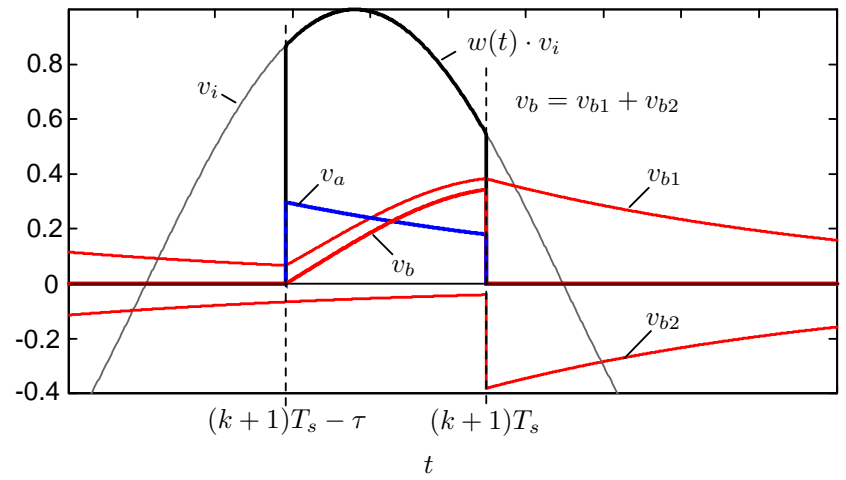

Fig. 8. $\quad v_{i}(t), w \cdot v_{i}(t)$ and various components of $v_{o n}(t)$.

$(k+1) T_{s}<t<(k+2) T_{s}-\tau^{\dagger}$. The memory effect of the capacitor, reflected as the non-zero output of path 61 in the interval $(k+1) T_{s}<t<(k+2) T_{s}-\tau$, should therefore be canceled. This can be achieved as follows. If we knew the output of path (61) at $t=(k+1) T_{s}$ (which we denote by $v_{b 1}(k+1)$ ), subtracting $v_{b 1}(k+1) e^{-\left(t-(k+1) T_{s}\right) / R C} u\left(t-(k+1) T_{s}\right)$ from the output of (b1) will result in path (b) having a zero output for the intervals $(k+1) T_{s}<t<(k+2) T_{s}-\tau$. This is due to the following. At $t=(k+1) T_{s}$, the input to the filter (with impulse response $(1 / R C) h(t)$ in Fig. 7(c)) goes to zero, and $v_{b 1}(k+1)$ will decay exponentially with a time-constant $R C$.

How do we determine $v_{b 1}(k+1)$ ? Just when the switch is turned on, the capacitor voltage is $v_{k}$ due to the past input memorized on the capacitor. When the switch is turned off at a time $\tau$ later, this voltage will have decayed to $v_{k} e^{-\tau / R C} \equiv \beta v_{k}$. At the same time, the output due to $w \cdot v_{i}(t)$ alone, which is the output of path (61) is $v_{b 1}(k+1)$. By linearity, the capacitor voltage $v_{o}$ at $(k+1) T_{s}$, therefore, has to be $\beta v_{k}+v_{b 1}(k+1)$. However, we know that, by definition, the capacitor voltage at $(k+1) T_{s}$ is $v_{k+1}$. Thus, $v_{b 1}(k+1)=v_{k+1}-\beta v_{k}$. From the discussion in the previous paragraph, we should subtract $v_{b 1}(k+1) e^{-\left(t-(k+1) T_{s}\right) / R C} u\left(t-(k+1) T_{s}\right)$ from the output of path (61) so that $v_{o n}(t)=0$ for $(k+1) T_{s} \leq t<(k+2) T_{s}-\tau$. This can be accomplished by exciting a filter with impulse response $h(t)$ with $\left(v_{k+1}-\beta v_{k}\right) \delta\left(t-(k+1) T_{s}\right)$, as shown in path (62). As shown in Fig. 8, the output of path (2), namely $v_{b 2}$, is the negative of $v_{b 1}$ when the switch is off, i.e., for $(k+1) T_{s} \leq t<(k+2) T_{s}-\tau$. As a result, their sum is zero during that interval. $v_{o n}(t)$ is simply the sum $v_{a}+v_{b}=v_{a}+v_{b 1}+v_{b 2}$.

The complete output waveform of the switched-RC circuit, therefore, is the sum of $v_{o f f}(t)$, and $v_{o n}(t)$ which in turn is comprised of paths (a) and (b), as shown in Fig. 7(c).

From an input-output perspective, the signal flow diagram of Fig. 7(c) is equivalent to that of Fig. 17 in [5]. The two approaches are identical with respect to $v_{\text {off }}(t)$ and path @). The flow graph of [5], however, differs from that in Fig. 7(c) with respect to the output of path (b). In that reference, $v_{i}$ excites the filter with impulse response $(1 / R C) h(t)$. The filter's output is multiplied by $w(t)$, and an appropriate waveform is added to account for

${ }^{\dagger} v_{\text {off }}(t)$, which is non-zero only during $(k+1) T_{s}<t<(k+2) T_{s}-\tau$, models that part of the waveform. initial conditions. In the opinion of the authors, the flow graph of Fig. 7(c) is more intuitively appealing, since in physical terms, the input excites the RC-network only when the switch is closed, i.e., $w(t)=1$.

In [4], Soer et. al propose a model for the output of the switched-RC network. This model, derived using state-space and frequency domain methods, appears (very) different. It turns out that it is equivalent to the model of Fig. 7(c), though the equivalence is not apparent. Soer's model looks much simpler that of Fig. 7(c). In the subsection that follows, we demonstrate that our signal-flow diagram is equivalent to that of Soer's model. We also show that there is a price to be paid for the apparent simplicity of that model.

\section{A. Connection to Soer's Model}

Observing Fig. 7(c) shows that the contributions of the $\beta$ paths in (a) and (b) are equal and opposite, and therefore cancel. Combining the remaining portions of paths (a) and (b) and recognizing that the $\mathrm{ZOH}$ can be represented as an integrator cascaded with a filter of impulse response $\delta(t)-\delta\left(t-\left(T_{s}-\tau\right)\right)$, we obtain the simplified signal flow graph of Fig. 9(a). Path (1) generates $v_{\text {off }}(t)$, while the sum of paths (2) and (3) yield $v_{\text {on }}(t)$. Combining the outputs of (1) and (2) of this figure leads to the signal flow graph in part (b). While this flow graph looks simpler, observe that the separation between $v_{\text {off }}(t)$ and $v_{\text {on }}(t)$ is lost.

The impulse response of the integrator is the unit step $u(t)$, and $h(t)=e^{-t / R C} u(t)$. Thus, their difference (which forms the input of the upper path in Fig. 9(b)) is $\left(1-e^{-t / R C}\right) u(t)$. This is immediately recognized as being the step-response of an RC-filter with impulse response $(1 / R C) h(t) u(t)$. This can be represented as a cascade of an integrator and a filter whose impulse is $(1 / R C) h(t)$, as shown in Fig. 9(c). Reordering the blocks of the top-most path, we obtain the signal-flow diagram of Fig. 9(d). The final step is to recognize the $\mathrm{ZOH}$ of width $\left(T_{s}-\tau\right)$ in this figure, and move the summer before the filter, yielding Fig. 9(e), which is Soer's model [4].

While Soer's model seems much simpler than that of Fig. 7(c) (and that shown in Fig. 17 in [5]), it makes it more difficult to understand the operation of the switched-RC kernel as part of an $N$-path filter, or in the passive-mixer mode. The primary reason for this is that attempting to merge the various paths in Fig. 7(c), while yielding a simpler looking signal-flow diagram, also makes it difficult to separate $v_{\text {off }}(t)$ and $v_{o n}(t)$ (which is needed to analyze the operation as an $N$-path filter), or identify the key constituents of the output in the passive-mixer mode.

\section{B. Operation as an N-Path Filter}

When the network of Fig. 1 is used as an $N$-path filter, the output is taken at $v_{x}$. Clearly, $v_{x}(t)$ is simply the sum of the $v_{o n}$ waveforms of the individual kernels. The signal-flow diagram that yields $v_{o n}(t)$ can be obtained from that in Fig. 7(c) by eliminating the path that yields $v_{\text {off }}$ and simplifying the remainder of the diagram. The result is shown in Fig. 10. When $v_{i}=e^{j 2 \pi f t}$, the component of $v_{o n}(t)$ at the frequency $f$ is comprised of 
(a)

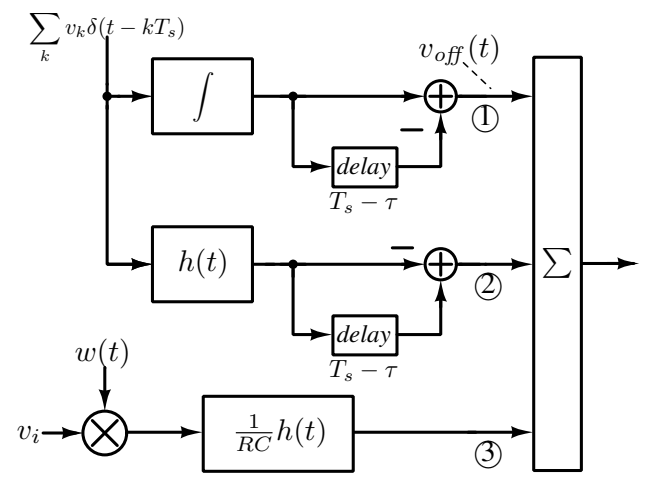

(b)

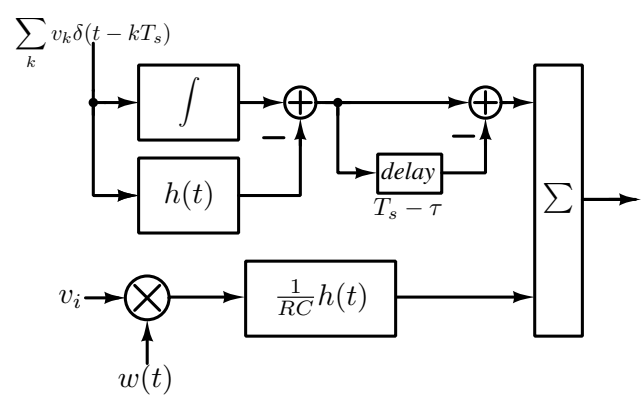

$\sum_{k}$
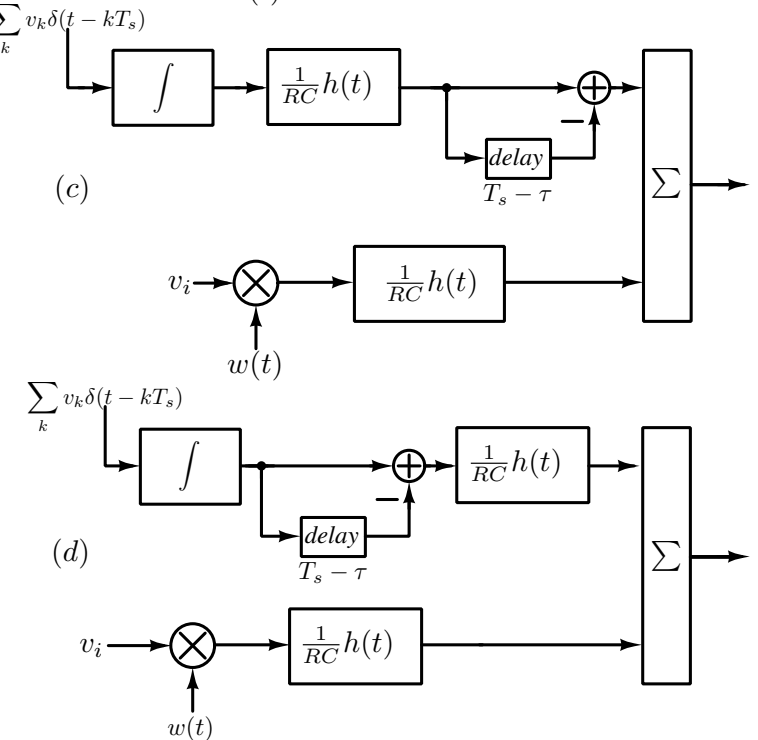

(e)

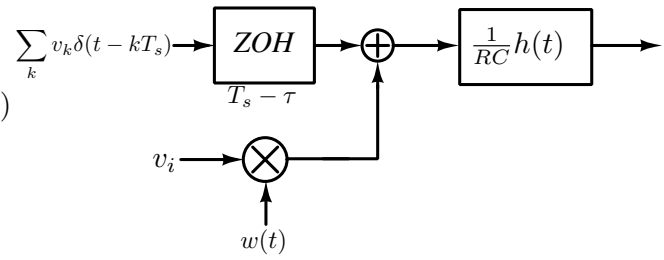

Fig. 9. Connection with Soer's model.

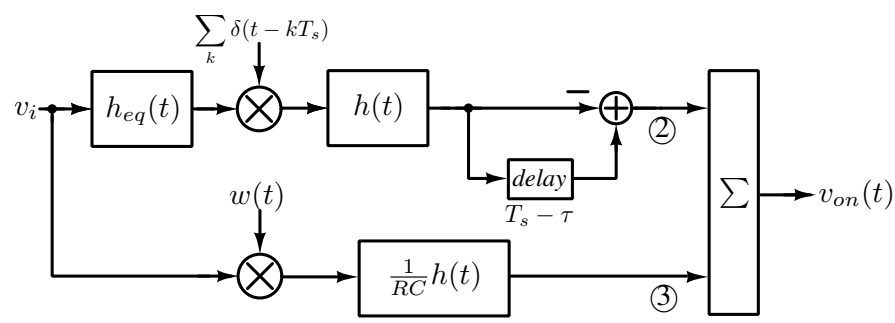

Fig. 10. Model of the switch-RC kernel needed to determine $v_{\text {on }}(t)$, which is relevant when operating the structure of Fig. 1 as an $N$-path filter. contributions from paths (2) and (3) as follows.

$$
\begin{gathered}
\text { Path (2) } \rightarrow-\underbrace{\frac{H_{e q}(f)}{T_{s}}}_{\begin{array}{c}
\mathcal{F}\left(h_{\text {eq }}(t)\right) \\
\text { and sampling }
\end{array}} \underbrace{\frac{R C}{1+j 2 \pi f R C}}_{\mathcal{F}(h(t))}\left(1-e^{-j 2 \pi f\left(T_{s}-\tau\right)}\right) \\
\text { Path (3) } \rightarrow \underbrace{\frac{\tau}{T_{s}}}_{\begin{array}{c}
\text { dc } \\
\text { component } \\
\text { of } w(t)
\end{array}} \cdot \underbrace{\frac{1}{1+j 2 \pi f R C}}_{\mathcal{F}(h(t) / R C)} .
\end{gathered}
$$

Thus, $X_{0}(f)$, the zeroth-order harmonic transfer function of the $N$-path filter, given by $N \cdot V_{x}(f) / e^{j 2 \pi f t}$, and using $N \tau=T_{s}$, is seen to be

$$
X_{0}(f)=\frac{1}{(1+j 2 \pi f R C)}\left[1-\frac{R C}{\tau} H_{e q}(f)\left\{1-e^{-j 2 \pi f\left(T_{s}-\tau\right)}\right\}\right] .
$$

Fig. 11 compares the magnitudes of simulated and analytical

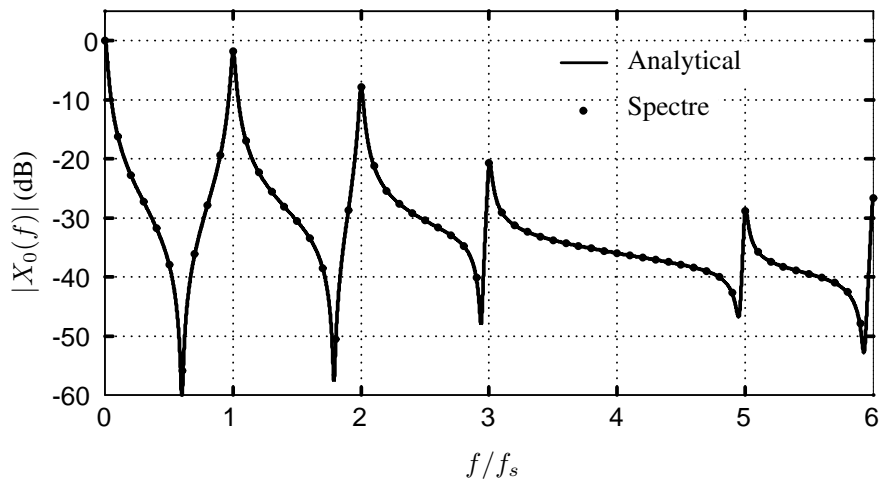

Fig. 11. Simulated and analytical $X_{0}(f)$ for $N=4, R=50 \Omega, C=50 \mathrm{pF}$ and $f_{s}=1 \mathrm{GHz}$.

$X_{0}(f)$ for $N=4, R=50 \Omega, C=50 \mathrm{pF}$ and $f_{s}=1 \mathrm{GHz}$.

For input frequencies around $f_{s}$, and $R C \gg T_{s}, X_{0}(f)$ can be simplified, using $2 \pi f R C \approx 2 \pi f_{s} R C \gg 1$ and $\tau / T_{s}=$ $1 / N \equiv D$, as follows ${ }^{\dagger}$.

$$
X_{0}(f) \approx \frac{H_{e q}(f)}{j 2 \pi f_{s} \tau}\left(e^{j 2 \pi f_{s} \tau}-1\right)=H_{e q}(f) e^{j \pi D} \operatorname{sinc}(D) .
$$

As seen from the discussion above, the transfer function of the equivalent LTI filter has a central role to play in our analysis of the $N$-path filter output.

\section{Input Admittance:}

Let the $N$-path circuit be driven by a voltage $v_{i}(t)=$ $e^{j 2 \pi\left(f_{s}+\Delta f\right) t}$, where $\Delta f \ll f_{s}$. The fundamental component of the current drawn from the source for input frequencies around $f_{s}$ is simply the component of the voltage across the resistor (at the input frequency) divided by $R$, and is given by

$$
I(f)=\frac{e^{j 2 \pi\left(f_{s}+\Delta f\right) t}}{R}\left(1-X_{0}(f)\right) .
$$

For $R C \gg \tau$ and $f \approx f_{s},(12)$ can be simplified as

$$
H_{e q}(f) \approx \frac{\operatorname{sinc}(D) e^{-j \pi D}}{(1+j 2 \pi \Delta f N R C)} .
$$

\footnotetext{
${ }^{\dagger}$ In this paper, we use the normalized sinc function, i.e., $\operatorname{sinc}(x)=\frac{\sin (\pi x)}{\pi x}$
} 
Using this in (19), we have

$$
I(f) \approx \frac{e^{j 2 \pi\left(f_{s}+\Delta f\right) t}}{R}\left(1-\frac{\operatorname{sinc}^{2}(D)}{1+j 2 \pi \frac{R C}{D} \Delta f}\right)
$$

The admittance, therefore, is

$$
Y(j 2 \pi(f+\Delta f)) \approx \frac{1}{R}\left(1-\frac{\operatorname{sinc}^{2}(D)}{1+j 2 \pi \frac{R C}{D} \Delta f}\right)
$$

In continued fraction form, the above expression can be written as

$$
Y=\frac{1}{R+\frac{1}{\frac{1-\operatorname{sinc}^{2}(D)}{R \cdot \operatorname{sinc}^{2}(D)}+j \frac{2 \pi \Delta f C}{D \cdot \operatorname{sinc}^{2}(D)}}}
$$

This is readily identified with the network shown in Fig. 12, in agreement with [6], [8] $]^{\dagger}$. The controlled source is necessary due to the following. The input has a frequency $\left(f_{s}+\Delta f\right)$. The current flowing through $C_{2}$ in the network of Fig. 12 would be proportional to $\left(f_{s}+\Delta f\right)$. However, as seen from the discussion above, the current is seen to be dependent only on $\Delta f$. The controlled source, therefore, is necessary to remove the current component at $f_{s}$.

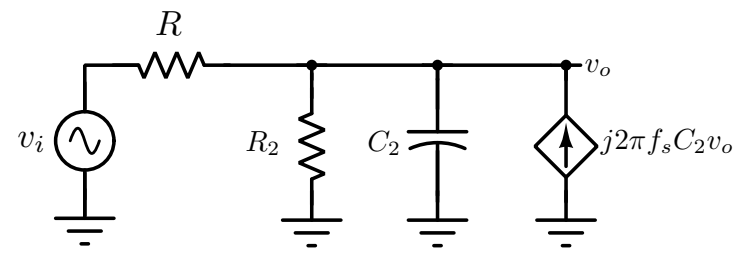

$$
R_{2}=R \frac{\operatorname{sinc}^{2}(D)}{1-\operatorname{sinc}^{2}(D)} \quad C_{2}=\frac{C}{D \operatorname{sinc}^{2}(D)}
$$

Fig. 12. Approximate LTI network for frequencies close to $f=f_{s}$.

\section{High-order Harmonic Transfer Functions:}

Higher-order harmonic transfer functions can be similarly determined. Thanks to $N$-path operation, only the $N^{t h}$ order HTFs and their multiples will be non-zero. Referring to Fig. 10, every $N^{t h}$ coefficient of the Fourier expansion of $w(t)$ is zero, since $w(t)$ has a width $T_{s} / N$. As a result, path (3) does not contribute to $H_{ \pm 4}$. We now determine $H_{-4}$. We assume that the input frequency is expressed as $4 f_{s}+f$. This way of denoting the input frequency is convenient, since the output frequency associated with $H_{-4}$ is then $\left(4 f_{s}+f\right)-4 f_{s}=f \cdot v_{i}=$ $e^{j 2 \pi\left(f+4 f_{s}\right) t}$ is filtered by the LTI filter with frequency response $H_{e q}$, before being sampled by the impulse train. The (complex) sinusoid at the sampler's input is $H_{e q}\left(f+4 f_{s}\right) e^{j 2 \pi\left(f+4 f_{s}\right) t}$. Sampling translates this sinusoid to all frequencies shifted by integer multiples of $f_{s}$. Since we are interested in the output frequency $f$, the output of the sampler at frequency $f$ is of interest. Clearly, this is $\left(1 / T_{s}\right) H_{e q}\left(f+4 f_{s}\right) e^{j 2 \pi f t}$. Denoting the Fourier transform of $h(t)$ by $H(f)$, the output of path (2) at $f$ is seen to be $\left(1 / T_{s}\right) H_{e q}\left(f+4 f_{s}\right) H(f)\left(1-e^{-j 2 \pi f\left(T_{s}-\tau\right)}\right) e^{j 2 \pi f t}$,

\footnotetext{
${ }^{\dagger}$ Unlike in a LTI network, we note that impedance and admittance are not reciprocals of each other
}

where $H(f)=R C /(1+j 2 \pi f R C)$. To determine $H_{4}$, we denote the input tone by $\left(-4 f_{s}+f\right)$ and proceed in a similar fashion. We thus have

$$
\begin{aligned}
& H_{-4}\left(4 f_{s}+f\right)=H_{e q}\left(4 f_{s}+f\right) \cdot H(f)\left(1-e^{-j 2 \pi f\left(T_{s}-\tau\right)}\right) \\
& H_{4}\left(-4 f_{s}+f\right)=H_{e q}\left(-4 f_{s}+f\right) \cdot H(f)\left(1-e^{-j 2 \pi f\left(T_{s}-\tau\right)}\right)
\end{aligned}
$$

The above expressions are in excellent agreement with simulations, as shown in Fig. 13. From the discussion above, we see

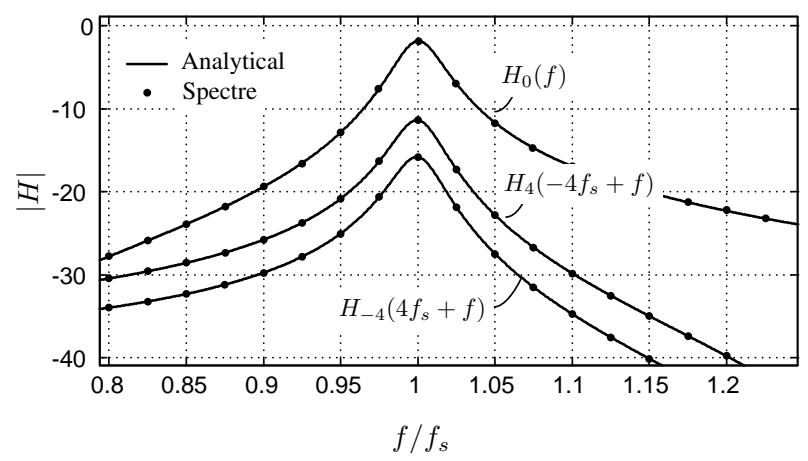

Fig. 13. Analytical and simulated $\left|H_{0}(f)\right|, H_{-4}\left(4 f_{s}+f\right), H_{4}\left(-4 f_{s}+f\right)$ for a 4-path filter: $R=50 \Omega, C=50 \mathrm{pF}, f_{s}=1 \mathrm{GHz}$.

that the folding of signals from higher frequencies into the desired band is largely controlled by the shape of $H_{e q}$, (again) placing in evidence the important role played by the voltages sampled on the capacitors.

\section{E. Simplified Model in the Passive-Mixer Mode}

(a)
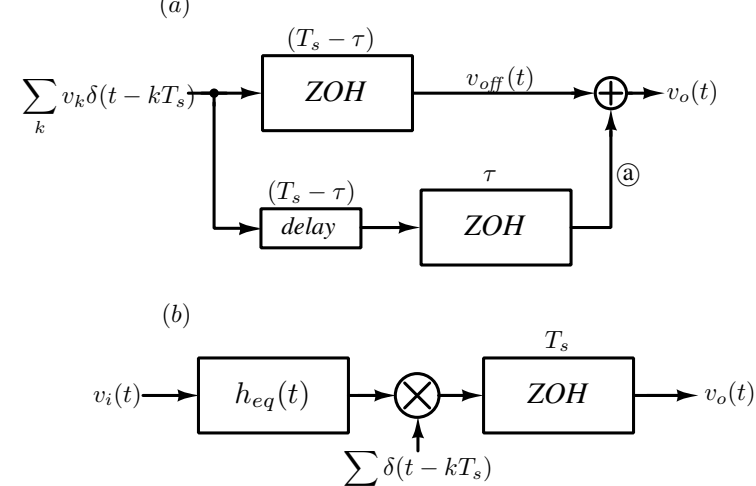

Fig. 14. (a) Simplified model in the passive-mixer mode and (b) Final model for the passive mixer.

In the passive-mixer (P-M) mode, $f_{i n} \approx f_{s}+\Delta f, R C \gg T_{s}$ and $f_{\text {in }} \gg 1 / R C$. The output voltage of Fig. 7(c) can then be simplified by recognizing the following. The output of path (b) is very small, since the input frequency is very large in relation to the bandwidth of the RC filter. Further, $h(t)$ is almost a unitstep function, since $R C \gg T_{s}$, and $\beta \approx 1$. As a result, $h(t)$ and the subsequent block in path (a) can be approximated by a $\mathrm{ZOH}$, with initial delay of $\left(T_{s}-\tau\right)$ and width $\tau$, as shown in Fig. 14(a). This is readily simplified as a single $\mathrm{ZOH}$ with width $T_{s}$. Thus, as concluded in [5], the performance in the P-M mode is dependent mostly on the sampled voltage across the capacitor. Note that such a simplification is not immediately apparent from 
Soer's model, or from the model of Fig. 9(a). Fig. 14(c) shows the simplified model of the switched-RC circuit in the P-M mode, including the prefilter.

Let the switched-RC circuit be operating in the P-M mode, with $v_{i}(t)=e^{j 2 \pi\left(m f_{s}+\Delta f\right) t}$, where $m$ is an integer and $\Delta f \ll f_{s}$. $1 /(2 \pi N R C)$ should be chosen so that $\Delta f$, which is the desired baseband tone, suffers very little attenuation.

Referring to Fig. 14(b), the output of the prefilter is $H_{e q}\left(m f_{s}+\Delta f\right) e^{j 2 \pi\left(n f_{s}+\Delta f\right) t}$. Due to impulse sampling and the subsequent hold operation, the desired output tone has an amplitude $H_{e q}\left(m f_{s}+\Delta f\right)$. In a down-conversion mixer $m$ is typically chosen to be unity. Using (12), it is easy to show that the ratio of the conversion gain from an undesired frequency $\left(m f_{s}+\Delta f\right), m \neq 1$ to that from the desired input at frequency $\left(f_{s}+\Delta f\right)$ can be approximated as

$$
\left|\frac{H_{e q}\left(m f_{s}+\Delta f\right)}{H_{e q}\left(f_{s}+\Delta f\right)}\right| \approx\left|\frac{\operatorname{sinc}(m D)}{\operatorname{sinc}(D)}\right| .
$$

\section{KERNEL NOISE}
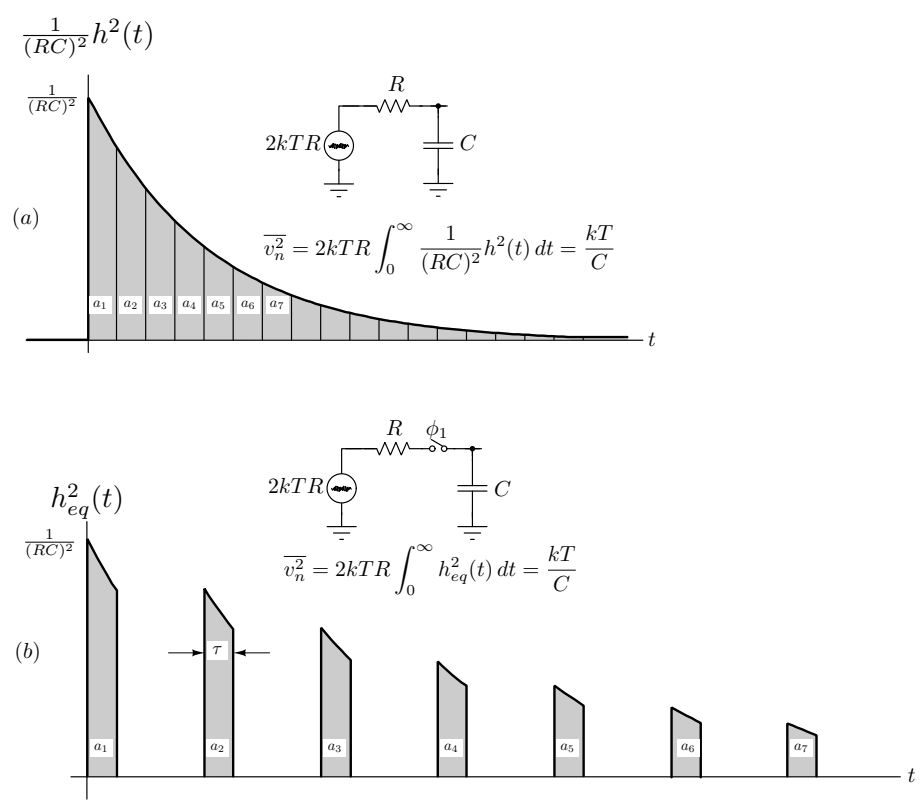

Fig. 15. (a) Mean square noise of the capacitor voltage, which is proportional to the integral of the square of the impulse response, is $k T / C$. (b)

In this section, we address kernel noise. First consider the LTI network of Fig. 15(a). The impulse response corresponding to the transfer function from the noise source (with a double-sided voltage noise spectral density of $2 k T R$ ) to the capacitor voltage is $(1 / R C) e^{-t / R C} u(t)=(1 / R C) h(t)$. The mean-square noise across the capacitor can be obtained using Parseval's theorem as follows.

$$
\begin{aligned}
\overline{v_{n}^{2}} & =2 k T R \int_{-\infty}^{\infty}|H(f)|^{2} d f \\
& =2 k T R \int_{0}^{\infty} \frac{1}{(R C)^{2}}|h(t)|^{2} d t=\frac{k T}{C} .
\end{aligned}
$$

Clearly, the mean square noise is proportional to the area under the squared impulse response.
When the capacitor is periodically switched, as in Fig. 15(b), the mean square noise across the capacitor is given by

$$
\overline{v_{n}^{2}}=2 k T R \int_{0}^{\infty}\left|h_{e q}(t)\right|^{2} d t .
$$

In Section III, we determined $h_{e q}(t)$ by injecting a current impulse into the capacitor of the adjoint network, and observing the current in the resistor. There, we saw that the capacitor voltage decays exponentially with a time-constant $R C$ when the switch is closed, and remains unchanged when the switch is open. As a result, the resistor current, which is $h_{e q}(t)$, decays exponentially for $0 \leq t<\tau$. At $t=T_{s}$, when the switch is closed again, $h_{e q}(t)$ begins from "where it left off", and decays again. One can therefore think of $h_{e q}^{2}(t)$ as being the result of cutting up the squared impulse response of Fig. 15(a) into slivers of width $\tau$, and spacing them $T_{s}$ apart. Thus, the area under the squared impulse response $h_{e q}^{2}(t)$ is exactly the same as that in Fig. 15(a). Therefore, the mean square noise across the capacitor in the switch-RC kernel is (not surprisingly) $k T / C$, irrespective of the sampling or mixing mode of operation and independent of the duty cycle. This result is identical to that in [4] (and [6])- where it was derived using frequency domain methods. It is thus seen that working in the time-domain yields insights that are less apparent in the frequency domain.

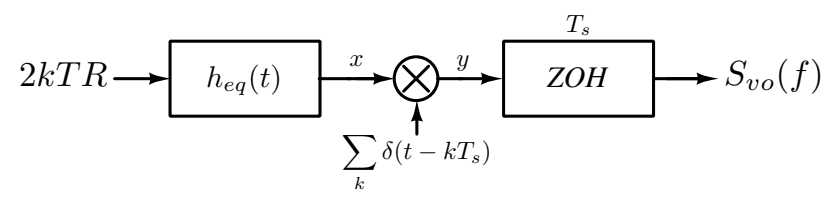

Fig. 16. Determining the output noise spectral density in the passive-mixer mode.

Next, we determine the noise spectral density of the switch$\mathrm{RC}$ kernel in the passive-mixer mode. The signal flow diagram is shown in Fig. 16. The double-sided noise spectral density due to the resistor is $2 k T R$. The resistor noise is filtered by an LTI filter with impulse response $h_{e q}(t)$ and sampled, before exciting a $\mathrm{ZOH}$ (which holds for a duration $T_{s}$ ). The autocorrelation function of the noise at $x$ is given by

$$
R_{x x}(t)=2 k T R \cdot h_{e q}(t) * h_{e q}(-t)
$$

where $*$ denotes convolution. Due to sampling, the autocorrelation function of the noise at $y$ is therefore

$$
R_{y y}(t)=\sum_{l=-\infty}^{\infty} R_{x x}\left(l T_{s}\right) \delta\left(t-l T_{s}\right) .
$$

The noise spectral density of the output waveform, denoted by $S_{v o}(f)$, is thus given by

$$
S_{v o}(f)=\underbrace{\left(\frac{1}{T_{s}} \sum_{l=-\infty}^{\infty} R_{x x}\left(l T_{s}\right) e^{-j 2 \pi f l T_{s}}\right)}_{S_{y y}(f)=\mathcal{F}\left(R_{y y}(t)\right)} \overbrace{T_{s}^{2} \operatorname{sinc}^{2}\left(f T_{s}\right)}^{Z O H} .
$$

In a down-conversion mixer, the spectral density around $f=0$ is of interest. This is given by

$$
S_{v o}(0)=T_{s} \sum_{l=-\infty}^{\infty} R_{x x}\left(l T_{s}\right)
$$


We therefore need to compute the sum of the samples of $R_{x x}(t)$, sampled at $f_{s} . R_{x x}(0)$ is simply the mean-square noise across the capacitor, and as we saw earlier in this section, is given by $k T / C$. $R_{x x}\left(T_{s}\right)$ is proportional to the area under $h_{e q}(t) \cdot h_{e q}\left(t-T_{s}\right)$ : observing Fig. 4(c), it is easy to see that $R_{x x}\left(T_{s}\right)=\beta R_{x x}(0)$. Proceeding similarly, we can write

$$
R_{x x}\left(l T_{s}\right)=\frac{k T}{C} \beta^{|l|}
$$

Thus,

$$
\sum_{l=-\infty}^{\infty} R_{x x}\left(l T_{s}\right)=\frac{k T}{C}\left(\frac{1+\beta}{1-\beta}\right) .
$$

In the passive-mixer mode, $\beta=e^{-\tau / R C} \approx 1-(\tau / R C)$. Using the equation above in (32), we obtain

$$
S_{v o}(0) \approx 2 k T R N
$$

which is identical to the result obtained in eq.52 of [4] and eq.21 of [6] using frequency-domain methods. Thanks to the adjoint impulse-response method, the determination of noise is considerably less involved than that in [4], [6].

\section{CONCLUSIONS}

The operation and properties of switched-RC $N$-path filters, samplers and passive mixers are intimately tied to the sampled voltages across the capacitors. In this work we exploited two key properties of sampled LPTV networks that greatly simplify the determination of the sampled capacitor voltages: first, that the sampled output of an LPTV network (when the sampling rate is the same as that at which the network is varying) can be thought of as the sampled output of an appropriately chosen linear time-invariant system. Next, the adjoint network can be used to determine the impulse response of the equivalent LTI system in a simple manner. We derived a model for the complete capacitor voltage in a switched-RC kernel. It turned out that this model was very convenient to understand the operation in the passive-mixer, sampler and $N$-path filter modes. Finally, we showed that noise analysis of the kernel, usually accomplished in the frequency domain, can also benefit from the simplicity of the adjoint-network approach.

\section{REFERENCES}

[1] A. A. Abidi, "The path to the software-defined radio receiver," IEEE Journal of Solid-State Circuits, vol. 42, no. 5, pp. 954-966, 2007.

[2] H. Darabi, A. Mirzaei, and M. Mikhemar, "Highly integrated and tunable RF front ends for reconfigurable multiband transceivers: A tutorial," IEEE Transactions on Circuits and Systems I: Regular Papers, vol. 58, no. 9, pp. 2038-2050, 2011.

[3] L. Franks and I. Sandberg, "An alternative approach to the realization of network transfer functions: The n-path filter," Bell Labs Technical Journal, vol. 39, no. 5, pp. 1321-1350, 1960.

[4] M. C. Soer, E. A. Klumperink, P.-T. De Boer, F. E. Van Vliet, and B. Nauta, "Unified frequency-domain analysis of switched-series- $R C$ passive mixers and samplers," IEEE Transactions on Circuits and Systems I: Regular Papers, vol. 57, no. 10, pp. 2618-2631, 2010.

[5] T. Iizuka and A. A. Abidi, "FET-RC Circuits: A unified treatment - Part I: Signal transfer characteristics of a single-path," IEEE Transactions on Circuits and Systems I: Regular Papers, vol. 63, no. 9, pp. 1325-1336, 2016.

[6] — - "FET-RC Circuits: A unified treatment - Part II: Extension to multipaths, noise figure, and driving-point impedance," IEEE Transactions on Circuits and Systems I: Regular Papers, vol. 63, no. 9, pp. 1337-1348, 2016.
[7] C. Andrews and A. C. Molnar, "A passive mixer-first receiver with digitally controlled and widely tunable RF interface," IEEE Journal of solid-state circuits, vol. 45, no. 12, pp. 2696-2708, 2010.

[8] _ - "Implications of passive mixer transparency for impedance matching and noise figure in passive mixer-first receivers," IEEE Transactions on Circuits and Systems I: Regular Papers, vol. 57, no. 12, pp. 3092-3103, 2010.

[9] A. Molnar and C. Andrews, "Impedance, filtering and noise in $N$-phase passive CMOS mixers," in Proceedings of the Custom Integrated Circuits Conference (CICC). IEEE, 2012, pp. 1-8.

[10] C. Andrews, C. Lee, and A. Molnar, "Effects of LO harmonics and overlap shunting on N-phase passive mixer based receivers," in Proceedings of the ESSCIRC (ESSCIRC). IEEE, 2012, pp. 117-120.

[11] D. Yang, C. Andrews, and A. Molnar, "Optimized design of N-phase passive mixer-first receivers in wideband operation," IEEE Transactions on Circuits and Systems I: Regular Papers, vol. 62, no. 11, pp. 2759-2770, 2015.

[12] A. Mirzaei, H. Darabi, J. C. Leete, and Y. Chang, "Analysis and optimization of direct-conversion receivers with $25 \%$ duty-cycle current-driven passive mixers," IEEE Transactions on Circuits and Systems I: Regular Papers, vol. 57, no. 9, pp. 2353-2366, 2010.

[13] A. Mirzaei and H. Darabi, "Analysis of imperfections on performance of 4phase passive-mixer-based high-Q bandpass filters in SAW-less receivers," IEEE Transactions on Circuits and Systems I: Regular Papers, vol. 58, no. 5, pp. 879-892, 2011.

[14] T. Strom and S. Signell, "Analysis of periodically switched linear circuits," IEEE Transactions on Circuits and Systems, vol. 24, no. 10, pp. 531-541, 1977.

[15] A. Ghaffari, E. A. Klumperink, M. C. Soer, and B. Nauta, "Tunable highQ N-path band-pass filters: Modeling and verification," IEEE Journal of Solid-State Circuits, vol. 46, no. 5, pp. 998-1010, 2011.

[16] A. Ghaffari, E. A. Klumperink, and B. Nauta, "Tunable N-path notch filters for blocker suppression: Modeling and verification," IEEE Journal of SolidState Circuits, vol. 48, no. 6, pp. 1370-1382, 2013.

[17] Z. Lin, P. I. Mak, and R. P. Martins, "Analysis and modeling of a gainboosted N-path switched-capacitor bandpass filter," IEEE Transactions on Circuits and Systems I: Regular Papers, vol. 61, no. 9, pp. 2560-2568, 2014.

[18] L. Duipmans, R. E. Struiksma, E. A. Klumperink, B. Nauta, and F. E. van Vliet, "Analysis of the signal transfer and folding in n-path filters with a series inductance," IEEE Transactions on Circuits and Systems I: Regular Papers, vol. 62, no. 1, pp. 263-272, 2015.

[19] S. Pavan and R. S. Rajan, "Interreciprocity in linear periodically timevarying networks with sampled outputs," IEEE Transactions on Circuits and Systems II: Express Briefs, vol. 61, no. 9, pp. 686-690, 2014.

[20] J. Vandewalle, H. De Man, and J. Rabaey, "The adjoint switched capacitor network and its application to frequency, noise and sensitivity analysis," International journal of circuit theory and applications, vol. 9, no. 1, pp. 77-88, 1981.

[21] F. Yuan and A. Opal, "Adjoint network of periodically switched linear circuits with applications to noise analysis," IEEE Transactions on Circuits and Systems I: Fundamental Theory and Applications, vol. 48, no. 2, pp. 139-151, 2001.

[22] R. Rohrer, L. Nagel, R. Meyer, and L. Weber, "Computationally efficient electronic-circuit noise calculations," IEEE Journal of Solid-State Circuits, vol. 6, no. 4, pp. 204-213, 1971.

[23] S. Pavan and R. S. Rajan, "Simplified analysis and simulation of the STF, NTF, and noise in continuous-time $\Delta \Sigma$ modulators," IEEE Transactions on Circuits and Systems II: Express Briefs, vol. 61, no. 9, pp. 681-685, 2014.

[24] A. Sukumaran and S. Pavan, "Design of continuous-time $\Delta \Sigma$ modulators with dual switched-capacitor Return-to-Zero DACs," IEEE Journal of SolidState Circuits, vol. 51, no. 7, pp. 1619-1629, 2016.

[25] S. Pavan, R. Schreier, and G. C. Temes, Understanding Delta-Sigma Data Converters, Second Edition. John Wiley \& Sons, 2016.

[26] L. Franks and I. Sandberg, "An alternative approach to the realization of network transfer functions: The $N$-path filter," Bell Labs Technical Journal, vol. 39 , no. 5, pp. 1321-1350, 1960. 


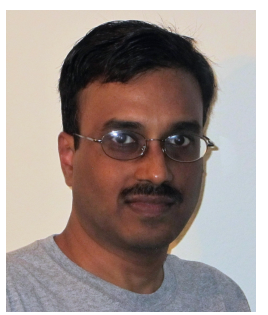

Shanthi Pavan obtained the B.Tech degree in Electronics and Communication Engg from the Indian Institute of Technology, Madras in 1995 and the M.S and Sc.D degrees from Columbia University, New York in 1997 and 1999 respectively. From 1997 to 2000, he was with Texas Instruments in Warren, New Jersey, where he worked on high speed analog filters and data converters. From 2000 to June 2002, he worked on microwave ICs for data communication at Bigbear Networks in Sunnyvale, California. Since July 2002, he has been with the Indian Institute of Technology-Madras, where he is now a Professor of Electrical Engineering. His research interests are in the areas of high speed analog circuit design and signal processing.

Dr. Pavan is the recipient of the IEEE Circuits and Systems Society Darlington Best Paper Award (2009), the Shanti Swarup Bhatnagar Award (2012) and the Swarnajayanthi Fellowship (2009) (from the Government of India), the Midcareer Research Excellence Award and the Young Faculty Recognition Award from IIT Madras (for excellence in teaching), the Technomentor Award from the India Semiconductor Association and the Young Engineer Award from the Indian National Academy of Engineering (2006). He is the author of Understanding Delta-Sigma Data Converters (second edition), with Richard Schreier and Gabor Temes). Dr. Pavan has served as the Editor-in-Chief of the IEEE Transactions on Circuits and Systems: Regular Papers, and on the editorial boards of both parts of the IEEE Transactions on Circuits and Systems. He has been on the technical program committee of the International Solid State Circuits Conference, and a Distinguished Lecturer of the Solid-State Circuits Society. He is a fellow of the Indian National Academy of Engineering.

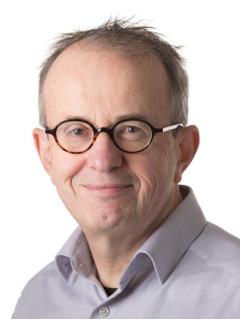

Eric Klumperink was born on April 4th, 1960, in Lichtenvoorde, The Netherlands. He received the B.Sc. degree from HTS, Enschede (1982), worked in industry on digital hardware and software, and then joined the University of Twente in 1984, shifting focus to ana$\log$ CMOS circuit research. This resulted in several publications and his Ph.D. thesis "Transconductance Based CMOS Circuits" (1997). In 1998, Eric started as Assistant Professor at the IC-Design Laboratory in Twente and shifted research focus to RF CMOS circuits (e.g. sabbatical at the Ruhr Universitaet in Bochum, Germany). Since 2006, he is an Associate Professor, teaching Analog \& RF IC Electronics, and guiding $\mathrm{PhD}$ and MSc projects related to RF CMOS circuit design with focus on software-defined radio, cognitive radio, and beamforming. $\mathrm{He}$ served as an Associate Editor for the IEEE Transactions on Circuits and Systems: Express Briefs (2006-2007), IEEE Transactions on Circuits and Systems: Regular Papers (2008-2009) and the IEEE Journal of Solid State Circuits(20102014), as IEEE Solid-State Circuit Society Distinguished Lecturer (2014/2015), and as member of the technical program committees of ISSCC (2011-2016) and the IEEE RFIC Symposium (2011-..). He holds several patents, authored and coauthored more than 150 internationally refereed journal and conference papers, and was recognized as 20+ ISSCC paper contributor over 1954-2013. He is a co-recipient of the ISSCC 2002 and the ISSCC 2009 "Van Vessem Outstanding Paper Award". 